\title{
Blind Manuscript Submission to Reduce Rejection Bias?
}

\author{
Khaled Moustafa ${ }^{i}$
}

High percentages of submitted papers are rejected at editorial levels without offering a second chance to authors by sending their papers for further peer-reviews. In most cases, the rejections are typical quick answers without helpful argumentations related to the content of the rejected material. More surprisingly, some journals vaunt their high rejection rates as a "mark of prestige"!

However, journals that reject high percentages of submitted papers have built their prominent positions based on a flawed measure, the impact factor, and from a long and favorable historical context. Their shareholders may think that they are allowed to have a large margin of rejection rates without affecting their sponsorship or funding sources thanks to an extended anchorage since tens, or in some cases hundreds, of years compared to unknown or new journals that struggle to pave a way in the scientific publication world. Historical anchorage of some journals also makes it unfair to compare old and new journals in term of whatever "popularity" or any ranking system. It will thus be unfair and biased appraisal to compare a journal that was launched in 2000 with a journal that was established in 1950 or 1900 or earlier.

Rejecting a high percentage of papers became an objective per se for elitist journals to take pride in an artificial elitist club, arguing strangely that a high rejection rate is a gauge of quality. Worse, sometimes rejection decisions are made after long months of waiting, upwards of a year in some cases without giving information about why the paper is rejected or how to improve it, for at least to compensate authors by useful advices for their wasted time in waiting the journal's negative decision. Doing so, they corrupt science and give negative images of their journals. Elitism is an attitude incompatible with science and knowledge dissemination.

Another subjective and unfounded rejection argumentation is based on "space limitation", as if a journal was a means of transport in which all places have been reserved and there are no more places available neither in the current or subsequent issues.

In order to reduce such biases and maintain the integrity of scientific publication, an important reform at the submission level would be required. Scientists need to be evaluated by peers based on the content of their articles, not on their nominal or institutional reputation. Editors are ultimately humans. They could be biased consciously or unconsciously in favor or against an article when they recognize the author's name, his gender, country or institution etc. In a recent report for example, it was shown that some biases relating to gender favoritism exist at faculty level (Moss-Racusin et al. 2012). In the same way, an article can be rejected upon initial screening without further consideration just because an editor has an impression about the 
author's name, his gender, his previous work, his country or his affiliated institution, despite the potential merit of the submitted material. Moreover, at a comparable level of a paper's contents, known scientists having track records and working at "prestigious institutions" or in a developed country would have much more of chance to get their papers accepted in the "highest ranked journal" than unknown scientists working in a developing country or at small or unknown institutions. English native speakers or countries would also have more chance and facility than non-native speakers or countries.

Blind submission (submission without disclosing the author's identity and contact details) would thus be an effective solution to reduce rejection biases at the editorial levels and the first stage of paper assessment. Anonymous submission will allow judging articles based only on their content and merit, far from any personal or institutional biases. Together with blind peerreview, blind submission would enhance the scientific integrity and impartiality. The blind review system was introduced to increase the objectivity of the publication assessment (Kmietowicz 2008). But objectivity is also needed, and more importantly, at the first stage of the paper's evaluation, because high rejection rates (up to 90-95\% in some cases) are made at this stage without "valid" reasons. Too much transparency may kill the transparency and the impartiality. When a double-blind review system was considered by an ecology journal, the representation of female authors was increased (Budden et al. 2008), suggesting that open review system has negative or biased effects on paper acceptances and rejections.

It is easily noticeable that there is an apparent asymmetry and double standard for paper acceptance and rejection policies. While the acceptance is generally decided at a collective level (editors plus reviewers), the rejection is often made by only one peer, the editor alone. To reject a paper, things are easy, but to accept a paper some editors need one or several reviewers' endorsements. This is a double standard that may need to be reformed because it means that the responsibility for paper acceptance is "higher" and "more serious" than the rejection's, though rejections often cause more moral damages for authors than acceptance for editors. A question may arise from this matter is: why are rejection and acceptance not treated equally?

One may also argue that a high rejection rate is sometimes unavoidable due to many more papers being received than the editors can handle. This may be true, particularly for "known" journals, which highlights another serious problem about why people prefer to target one specific journal rather than another. The answer is straightforward. Many authors believe that publishing in "high impact factor journals" will valorize them more than publishing in low impact factor journals. Although this is a highly questionable matter, it also poses the problem of the biased evaluation policy conducted by hiring or funding committees that rely on flawed ranking systems to evaluate people or to attribute funds. One of the reasons of the insistence to use such flawed measures (impact factor or similar measures) could be explained, at least partially, by the fact that it is easier for evaluators to compare simple numbers than to read longer texts to probe their potential values and make an objective assessment. For evaluators 
and administrators, who are in most cases non-scientists or scientists who were 'politicized' for administrative roles, it is easier and faster to compare abstract numbers (i.e. impact factor or number of publications) than written texts that need time, intellectual efforts and concentration to draw an objective assessment compared to a simple number that will do the job painlessly, just by its superior or inferior values. Additionally, the highest impact factor journals are generally Review Journals whose articles are mostly "commissioned" or "solicited", which adds a further layer of flaws to the impact factor and shows to which extent the impact factor could be handled (Moustafa 2014).

Publication is not a materialistic matter nor a commercial brand to be categorized into quality classes or ranks based on unique ingredients or exclusive characteristics that one brand can offer but not another. Publication, instead, is an intellectual contribution that could be conveyed in any form (printed or online) in any journal (old or new, with high or low impact factor). In other words, articles are peer-reviewed in the same way whatever the journal, and it does not make much sense that journal $X$ should be "more valuable" than journal $Y$. In a materialistic world, on other hand, there may be differences in ingredients or characteristics of manufactured goods, but in a scientific publication, journals fairly follow the same process and principles: peer-review followed by publication on paper and/or online, and authors have multiple choices to equally submit to either one. The same reviewers can also be reviewers for journal $X$ or $Y$, for high or low impact factor journals. Any notion of "superiority" should thus be meaningless, apart from the originality of the published thoughts or ideas independently from the journal name or its history or its managers.

At any rate, blind submission cannot be a rampart against high rejection rates but as a "buffer" to remove potentially subjective factors from the rejection decisions at the first assessment level. Editors can continue to reject high percentages of submitted papers, if they judge it necessary for their journals, but with a blind submission approach they will do it with greater objectivity and neutrality.

Some issues, however, may arise from a blind submission system. The first is the risk for a "selfpeer review". Since editors will not know the authors' identity, an author might be invited to judge his/her own paper. This issue, however, can be easily surmounted from a technical viewpoint. Regardless of the fact that the authors should be aware about such an eventuality, a technical solution could also be implemented to avoid such an issue before it happens. One simple code line introduced in the source code of the managing software can solve the problem; for example, If the name(s) of the invited reviewer(s) is/are identical to the author(s) name(s), then do not send the review invitation (or notify the editor...).

In a blind submission system, the communication with the corresponding authors can also be personalized by automatic association between the submission number and the author's name, as is the case of the acknowledgements sent automatically upon submissions. With the new facility of web-based technologies, the blind submission is relatively easy to implement and to operate for a better assessment of the scientific integrity and reliability. 
Since authors do not wish to damage editors or reviewers, a good option would be a submission system in which the author's identity should be undisclosed while the evaluator's identity (including editors and reviewers) should be disclosed. In such a system, editors and reviewers would be responsible for their decisions in case of any subjective assessment or personal biases. The authors, on the other hand, are already responsible for their publications (since their names will be shown), while the names of reviewers and editors are in most cases not disclosed. Undisclosed authors should not matter as the objective of the peer-review process is to objectively assess a manuscript, not the author's name.

Moreover, in a submission system of disclosed evaluators/undisclosed authors, reviewers will cease to be virtual numbers; authors will call them more professionally by their names rather than by numbers (reviewer 1 , reviewer 2...). Reviewers, on the other hand, will address their comments as to "the author(s)", without any particular issue.

Finally, if journals' editors want to maintain scientific integrity and protect their publication policy from any bias, they should innovate at the submission level and remove any partial or personal influences by adopting a system of undisclosed author/disclosed evaluators. Rejecting papers on subjective criteria may turn scientists away toward other alternatives or we may end with each scientist or institution having his or its journal. The rejection decisions should also be developed and founded on real helpful argumentations rather than on typical or superficial argumentations such as "space limitation" or "high pressure for space..." which do not make much sense. Authors would accept a rejection decision that is useful to improve their manuscript rather than to increase the "reject prestige" of the targeted journal, which will vaunt its amplified rejection rate as "further prestige". On other hand, most submission processes are currently long, daunting and time consuming. The submission process should be simplified and limited to only login and file uploading in the first stage. Upon acceptance, authors would be "happy" to provide all the required information and the specific page formatting. In such a case, the time devoted to answer a long series of questions and make a specific formatting is justified by the acceptance; in the case of rejection, it is not less than a waste of time for authors, editors and journals' managers.

\section{References}

Budden, A. E., Tregenza, T., Aarssen, L. W., Koricheva, J., Leimu, R., \& Lortie, C. J. (2008). Double-blind review favours increased representation of female authors. Trends in Ecology \& Evolution, 23(1), 4-6.

Kmietowicz, Z. (2008). Double blind peer reviews are fairer and more objective, say academics. British Medical Journal, 336(7638), 241. 
Moss-Racusin, C. A., Dovidio, J. F., Brescoll, V. L., Graham, M. J., \& Handelsman, J. (2012). Science faculty's subtle gender biases favor male students. Proceedings of the National Academy of Sciences of the United States of America, 109(41), 16474-16479.

Moustafa, K. (2014). The disaster of the impact factor. Science and Engineering Ethics. doi:10.1007/ s11948-014-9517-0.

'Email: khaled.moustafa@frenxiv.org 